\title{
Tradução, adaptação cultural e confiabilidade da versão em português brasileiro do questionário DRAM para avaliação psicométrica em dor lombar*
}

\section{Translation, Cross-cultural Adaptation and Reliability of Brazilian portuguese version of the DRAM Questionnaire for Psychometric Evaluation in Low Back Pain}

\author{
Carlos Tucci ${ }^{1}$ Alberto Jacob ${ }^{1}$ Thiago Bonato de Amorim ${ }^{1}$ Alex Oliveira de Araújo ${ }^{1}$ \\ Alexandre Fogaça Cristante ${ }^{1}$ \\ ${ }^{1}$ Instituto de Ortopedia e Traumatologia, Hospital das Clinicas, Faculdade \\ de Medicina, Universidade de Sao Paulo, Sao Paulo, SP, Brazil \\ Rev Bras Ortop 2020;55(1):54-61. \\ Endereço para correspondência Carlos Tucci, MD, MSc, Instituto de \\ Ortopedia e Traumatologia, Hospital das Clinicas HCFMUSP, \\ Faculdade de Medicina, Universidade de Sao Paulo, Rua Mato Grosso \\ 306, $1^{\circ}$ andar, São Paulo, SP, Brasil. CEP: 01239-040 \\ (e-mail: carlos.tucci@vita.org.br).
}

\section{Resumo \\ Objetivo A partir de estudos sobre a fisiologia da dor e suas relações com estados psicológicos, tornou-se essencial a avaliação psicológica dos indivíduos com quadros dolorosos, para selecionar os perfis mais favoráveis às diferentes formas de tratamento. O questionário Distress Risk Assessment Method (DRAM, na sigla em inglês) foi desenvol- vido como instrumento de triagem para portadores de dor na coluna vertebral subclassi- ficando os indivíduos em quatro grupos distintos (normal, sob risco, somático e depressivo), conforme a pontuação dos dois questionários que compõem o DRAM (Questionário Modificado de Percepção Somática [MSPQ, na sigla em inglês] e Zung). O objetivo do presente estudo é traduzir e adaptar o DRAM para o português brasileiro da versão original em inglês, além de analisar a confiabilidade da versão traduzida e adaptada. \\ Palavras-chave \\ - dor nas costas \\ - dor lombar \\ - doenças da coluna vertebral \\ - qualidade de vida \\ - resultado do tratamento \\ - medição de risco \\ - inquéritos e questionários \\ Método Segundo a metodologia International Quality of Life Assessment (IQOLA), consagrada em inúmeras publicações, foi desenvolvida uma versão em português brasileiro que foi aplicada a uma amostra de 85 indivíduos dos 3 centros participantes portadores de dor lombar. \\ Resultados Os resultados comprovaram a confiabilidade e reprodutibilidade da versão traduzida e adaptada do questionário DRAM com índice de Cronbach alfa de 0,815 para o MSPQ e de 0,794 para o Zung, e coeficiente de correlação intraclasse de 0,688 para o MSPQ e de 0,659 para o Zung. \\ Conclusão Tais dados permitiram concluir que a versão do questionário DRAM traduzida e adaptada culturalmente para o português brasileiro é confiável e está disponível para uso na prática clínica.}

\footnotetext{
Trabalho realizado no Instituto de Ortopedia e Traumatologia, Hospital das Clinicas HCFMUSP, Faculdade de Medicina, Universidade de São Paulo, São Paulo, SP, Brasil.
}

recebido

13 de Agosto de 2018 aceito

27 de Novembro de 2018
DOI https://doi.org/ 10.1055/s-0039-1700812. ISSN 0102-3616.
Copyright $(2020$ by Sociedade Brasileira License terms de Ortopedia e Traumatologia. Published by Thieme Revinter Publicações Ltda, Rio de Janeiro, Brazil 
Abstract

\section{Keywords}

- back pain

- low back pain

- spinal diseases

- quality of life

- treatment outcome

- risk assessment

- surveys and questionnaires
Objective Based on studies regarding pain physiology and its relation to emotional distress conditions, psychological evaluation became essential to determine the most favorable patient profiles to distinct therapeutic approaches. The Distress Risk Assessment Method (DRAM) has been developed as a screening instrument for patients with lumbar pain, classifying them in subgroups as normal, at risk, distressed somatic and distressed depressive, based on the two components of DRAM scores (Modified Somatic Perception Questionnaire [MSPQ] and Zung questionnaires). The objective of the present study is to translate and culturally adapt the DRAM to the Brazilian Portuguese language, and to determine the reliability of the final version.

Methods As proposed by the International Quality of Life Assessment (IQOLA) method, a Brazilian Portuguese version of the DRAM has been applied to a sample of 85 individuals from 3 participant centers.

Results The results confirmed the reliability and reproducibility of the DRAM in its Brazilian Portuguese final version: Cronbach alpha of 0.815 (MSPQ) and 0.794 (Zung) and intraclass correlation coefficient (ICC) of 0.688 (MSPQ) and 0.659 (Zung).

Conclusion The presented DRAM version in Brazilian Portuguese is reliable and is available to clinical practice use.

\section{Introdução}

Lombalgia é um fenômeno multidimensional, composto por fatores psicológicos (sensitivos, afetivos, cognitivos, comportamentais) e sociais. ${ }^{1,2}$ Os principais fatores de risco para incapacidade de curto e longo prazo em quadros de dor lombar inespecífica são psicossociais, e a remissão permanente dos quadros dolorosos está ligada a fatores ocupacionais e ao perfil psicométrico dos indivíduos avaliados. ${ }^{3}$ Ansiedade, depressão, hostilidade e outros traços de estresse psicológico são fatores que intervêm em vários desfechos em saúde, como a dor e a função. ${ }^{4,5}$

Devido à associação entre distúrbios psicológicos e desfechos das afecções da coluna vertebral, ${ }^{6,7}$ atualmente é aceito como ideal o modelo de abordagem biopsicossocial na avaliação inicial, tomada de decisão e avaliação final dessas doenças. Tal modelo requer instrumentos de avaliação psicométrica que resultam em escores que determinam, de diferentes maneiras, o estado psicológico do indivíduo avaliado. ${ }^{8-12}$

Revisões sistemáticas da literatura já mostraram a associação entre distúrbios psicológicos e a dor lombar, ${ }^{13}$ e revelaram que pacientes com depressão têm piores desfechos após a artrodese, ${ }^{14}$ e também que a terapia cognitiva comportamental pode reduzir a dor após a cirurgia. ${ }^{15}$ Se pacientes com diferentes características psicológicas sofrem de dor de forma diferente e obtêm diferentes resultados do tratamento cirúrgico, faz sentido compreender melhor essas características antes de indicar a cirurgia. Entre os inúmeros instrumentos psicométricos disponíveis na literatura atual, o Distress Risk Assessment Method (DRAM), ${ }^{16,17}$ desenvolvido originalmente para medir os estados psicológicos em indivíduos portadores de dor lombar, é uma ferramenta de avaliação psicológica a ser preenchida pelo paciente.

É de particular interesse, no método DRAM, a capacidade de identificar indivíduos de subtipos psicológicos distintos, em que características ansiosas (DS) ou depressivas (DD) podem se relacionar com comportamentos menos favoráveis a tratamentos cirúrgicos. O DRAM permite, portanto, identificar aqueles que necessitam de uma abordagem multidisciplinar, por apresentarem sinais de estresse psicológico com potencial de interferir nos sintomas de dor. É uma ferramenta simples e eficiente que permite alertar médicos cuidadores da necessidade de instituir uma abordagem psicológica conjunta ao diagnóstico e à indicação de tratamento médico.

Porém, o instrumento DRAM, originalmente desenvolvido em língua inglesa, não está disponível em versão validada para a língua portuguesa. O objetivo do presente estudo é criar uma versão do questionário DRAM, confiável e reprodutível, adaptada culturalmente à língua portuguesa falada no Brasil.

\section{Métodos}

Este é um estudo de tradução, adaptação cultural e análise da confiabilidade do questionário DRAM para a língua portuguesa falada no Brasil. O protocolo deste estudo foi avaliado e aprovado pelo Comitê de Ética em Pesquisa, conforme parecer consubstanciando o número 36615514.7.1001.0068. Todos os participantes assinaram termo de consentimento livre e esclarecido.

\section{Questionário DRAM}

O questionário é composto pela combinação dos resultados da pontuação de dois outros questionários: o Questionário Modificado de Percepção Somática (MSPQ na sigla em inglês $)^{17}$ e o Índice Modificado de Depressão de Zung (mZDI, na sigla em inglês). ${ }^{18} \mathrm{O}$ MSPQ é um questionário composto por 22 itens, com 4 alternativas possíveis para cada item, com pontuações de 0 (nunca), 1 (um pouco), 2 (bastante ou muito), 3 (demais, não poderia ser pior). Para a pontuação final, só são considerados os itens 2, 3, 7, 8, 9, 11, 13, 14, 16, $18,19,20,21$, como forma de confundir itens mais ou menos relavantes. A pontuação máxima varia de 0 a 39 pontos. 
O MSPQ foi desenvolvido especificamente para pacientes com dor nas costas e permite quantificar a percepção somática e autonômica, "ansiedade somática" ou "somatização". 0 mZDI, por sua vez, gradua o estado depressivo dos pacientes através da soma das pontuações de 0 a 3 das respostas para itens, com pontuação máxima de 69 .

A partir das pontuações obtidas nos questionários MSPQ e mZDI, o DRAM classifica os indivíduos em: Tipo N, normal, com escore do $\mathrm{mZDI}<17$ pontos, sem evidência de distúrbio psicológico ou de comportamento anormal com relação à doença; Tipo $\mathrm{R}$, em risco, escore do mZDI entre 17 e 33, e escore do MSPQ $<12$, mostrando predominantemente sintomas de depressão; Tipo $\mathrm{DD}$, depressivo, com escore do mZDI > 33; e Tipo DS somático/ansioso, escore do mZDI entre 17 e 33, MSPQ $>12$.

\section{Tradução e Adaptação do DRAM}

A fim de ser utilizado na prática clínica na população brasileira, o DRAM foi traduzido e adaptado culturalmente através da metodologia proposta pelo projeto International Quality of Life Assessment (IQOLA, na sigla em inglês), ${ }^{19}$ uma metodologia confiável, reprodutível e de aplicabilidade simples. No presente estudo, o método de tradução e adaptação, segundo a abordagem IQOLA adaptada pela Academia Americana de Cirurgiões Ortopedistas (AAOS, na sigla em inglês), ${ }^{20}$ seguiu um passo a passo simples e prático, conforme ilustrado na - Figura $\mathbf{1}$.

\section{Participantes}

O questionário DRAM traduzido foi aplicado a uma amostra de participantes para estudo de suas propriedades psicométricas, como consistência interna e reprodutibilidade. Os indivíduos recrutados para o presente estudo foram selecionados em ambulatórios de ortopedia e serviços de pronto-atendimentos em três diferentes cidades. Este estudo almejou 85 indivíduos, com base no universo amostral de trabalhos semelhantes. ${ }^{21-23}$

Critérios de inclusão: indivíduos > 18 anos; dor lombar inespecífica de qualquer duração (característica mecânica e postural); alfabetizado e fluente em língua portuguesa brasileira; disposto e capaz de emitir consentimento por escrito. Critérios de exclusão: sinal neurológico; histórico de trauma; cirurgia prévia na coluna lombar; língua nativa diferente do português brasileiro; doenças psiquiátricas diagnosticadas previamente; déficit cognitivo de qualquer grau; doença neurológica; presos ou reclusos em instituições correcionais.

\section{Aplicação da Versão Traduzida}

O questionário DRAM traduzido foi inicialmente aplicado a 30 indivíduos (piloto), quando então foi feita uma análise interina, e depois a mais 55, totalizando os 85. O DRAM é um

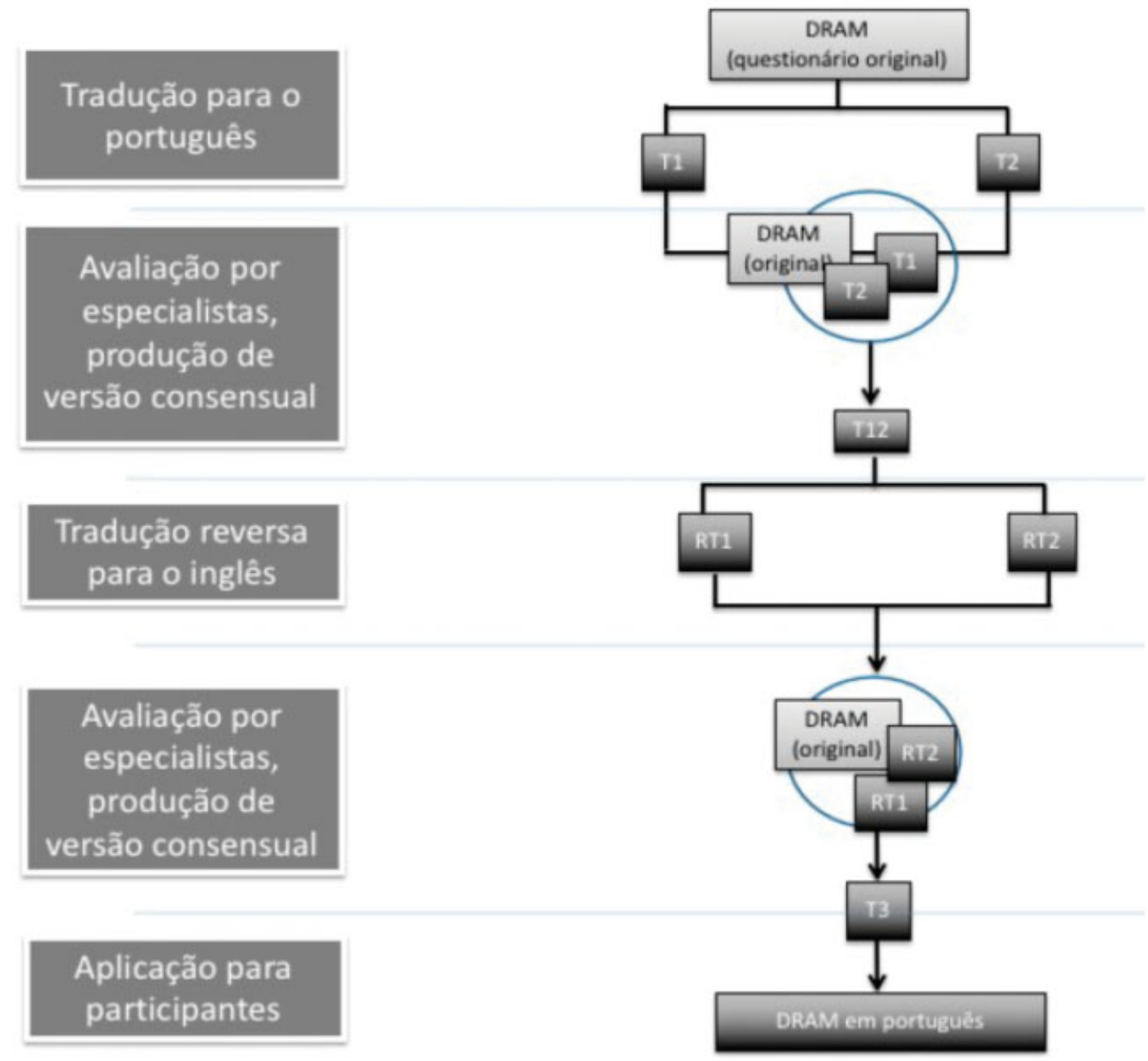

Fig. 1 Passos do método adotado para tradução adaptação. 
questionário autoaplicado; todos os pacientes incluídos neste estudo responderam os questionários sem qualquer intervenção do entrevistador. Aos primeiros 30 participantes, foi também aplicado um questionário adicional com as três perguntas abaixo, dando oportunidade e privacidade para dados qualitativos que pudessem revelar qualquer dificuldade com o uso do DRAM: (1) O Sr.(a). tem algum comentário sobre o idioma deste questionário? (2) Houve alguma pergunta ou item que não esteja claro ou compreensível em português? (3) O Sr.(a). gostaria de fazer alguma sugestão ou comentário sobre este questionário?

Finalizada essa sequência piloto de 30 aplicações, o autor principal tabulou os dados do DRAM para verificar se havia alguma inconsistência, e também analisou as respostas das três perguntas acima, para realizar alguma alteração ou correção no questionário traduzido, caso fosse necessário. Após a análise dos dados estudados nesse estágio, e como não foram detectados problemas, a amostra piloto foi integrada ao universo amostral total, e seguiu-se o estudo até completar a amostra de 85 pacientes.

Segundo preconizado pela metodologia IQOLA, ${ }^{19}$ os participantes responderam aos questionários em 2 situações distintas, com intervalos de 15 a 60 dias entre elas, o que corresponde ao tempo usual de intervalo entre consultas de pacientes com dor lombar. Assim, foi possível verificar a reprodutibilidade dos resultados do DRAM, conforme descrito adiante.

\section{Estudo das Propriedades Psicométricas do DRAM Traduzido}

As respostas individuais ao DRAM traduzido foram tabuladas em um banco de dados, por paciente, e também os escores totais. Seguiu-se uma análise da consistência interna do instrumento, utilizando-se o alfa de Cronbach. ${ }^{24}$ Este teste indica a homogeneidade dos fatores entre os itens dentro de um questionário ou subdomínios do questionário. $\mathrm{O}$ alfa de Cronbach também é usado para determinar a inter-relação entre os itens de um questionário. Um valor baixo indica baixa correlação entre itens projetados para medir o mesmo construto, enquanto um valor muito alto indica redundância entre um ou mais itens. Neste trabalho, o valor de referência adotado para indicar boa correlação foi entre 0,70 e $0,95 .^{25}$

Depois, foi analisada a reprodutibilidade dos dados contínuos do instrumento, utilizando-se o coeficiente de correlação intraclasse (CCI). O CCI é usado para medir a confiabilidade entre avaliadores para dois ou mais avaliadores que avaliaram o mesmo indivíduo. Pode ser usada para avaliar a confiabilidade teste-reteste. O CCI pode ser conceituado como a razão entre a variância entre grupos e a variância total. ${ }^{26}$

Os dados foram digitados, tabulados e checados em planilhas Microsoft Excel (Microsoft Corporation, Redmond, WA, EUA) pelo autor principal do presente estudo. A análise estatística foi realizada utilizando-se o software SPSS Statistics para Windows, Versão 12 (SPSS Inc. Chicago, IL, EUA).

\section{Resultados}

A metodologia de tradução e adaptação cultural proposta (IQOLA) eleita para o presente estudo mostrou-se prática e replicável. Os dois tradutores (um deles tradutor profissional juramentado e o outro profissional médico cirurgião de coluna fluente em língua inglesa) produziram independentemente versões compreensíveis e fiéis ao original, como comprovou o grupo de dois médicos e dois fisioterapeutas, ao sugerirem mínimas adequações na criação da versão T12 do questionário DRAM, assim como no desenvolvimento das versões retraduzidas.

Nessa fase, houve discussão pelo comitê de especialistas sobre as expressões coloquiais como feeling hot all over, sweat all over, stomach churning, butterflies in the stomach e desire to pass water, que foram comparadas às traduções propostas calor por todo o corpo, suado no corpo todo, estômago embrulhado, frio na barriga e vontade de urinar, e concluiu-se pela ausência de discrepâncias após consulta aos tradutores. A versão final, por sua vez, aplicada ao universo amostral inicial (piloto) de portadores de dor nas costas, mostrou-se compreensível e não foi alvo de comentários ou sugestões, como constatado pela aplicação de um "questionário sobre o questionário DRAM".

Após a análise dos dados estudados na etapa piloto, não foram detectadas alterações a serem realizadas. Assim, a amostra piloto foi integrada ao universo amostral total, e seguiu-se o estudo até completar a amostra total de pacientes.

De 108 pacientes inicialmente rastreados, 85 responderam integralmente os questionários no prazo estabelecido para as variáveis do escore MSPQ e do índice de depressão de Zung durante suas consultas ambulatoriais, sem que houvesse necessidade de alterar o plano de tratamento original, exceto pela adição do tempo de preenchimento dos documentos do estudo. Os demais 23 indivíduos foram excluídos por não terem respondido pela segunda vez os questionários ou por terem respondido fora do prazo estabelecido de 15 a 60 dias. A - Tabela 1 mostra a descrição das pontuações dos questionários nas duas ocasiões do preenchimento.

Dos 85 indivíduos incluídos, 54 (63,5\%) eram do sexo feminino, com idades entre 22 e 55 anos, com média de 31,2 anos de idade. Quanto à escolaridade, 40 (46,7\%) cursaram o primeiro grau completo, $37(43,5 \%)$ cursaram o segundo grau completo e $8(9,8 \%)$ tinham diploma universitário.

A consistência interna da versão final do questionário DRAM ( - Tabela 2) foi verificada pelo índice de Cronbach alfa resultante do presente estudo: 0,815 e 0,794, para MSPQ e Zung modificado, respectivamente.

Tabela 1 Escore do Questionário Modificado de Percepção Somática (MSPQ) e Índice Modificado de Depressão de Zung em suas primeira e segunda aplicações

\begin{tabular}{|l|l|l|}
\hline & Média & Desvio padrão \\
\hline MSPQ 1 & 11,6706 & 6,57446 \\
\hline MSPQ 2 & 10,0000 & 5,63577 \\
\hline Zung 1 & 24,9882 & 9,63562 \\
\hline Zung 2 & 23,0353 & 8,21359 \\
\hline
\end{tabular}

Abreviação: MSPQ, questionário modificado de percepção somática. 
58 Versão em português brasileiro do questionário DRAM Tucci Neto et al.

Tabela 2 Versão em português brasileiro do questionário DRAM

\begin{tabular}{|c|c|c|c|c|}
\hline \multicolumn{5}{|l|}{ MSPQ } \\
\hline \multicolumn{5}{|c|}{$\begin{array}{l}\text { Descreva como você se sentiu durante a SEMANA PASSADA assinalando com um }(\mathrm{X}) \text { na caixa apropriada. Responda todas as } \\
\text { perguntas. Não pense muito antes de responder. }\end{array}$} \\
\hline & Nunca & Um pouco & Bastante, muito & $\begin{array}{l}\text { Demais, não } \\
\text { poderia ser pior }\end{array}$ \\
\hline Aumento na frequência cardíaca & 0 & 1 & 2 & 3 \\
\hline Sensação de calor no corpo todo & 0 & 1 & 2 & 3 \\
\hline Suado no corpo todo & 0 & 1 & 2 & 3 \\
\hline Suado em uma parte específica do corpo & 0 & 1 & 2 & 3 \\
\hline Palpitação nas veias do pescoço & 0 & 1 & 2 & 3 \\
\hline Dor de cabeça latejante & 0 & 1 & 2 & 3 \\
\hline Tontura & 0 & 1 & 2 & 3 \\
\hline Visão embaçada & 0 & 1 & 2 & 3 \\
\hline Sensação de desmaio & 0 & 1 & 2 & 3 \\
\hline Sensação de que tudo parece irreal & 0 & 1 & 2 & 3 \\
\hline Náusea & 0 & 1 & 2 & 3 \\
\hline Sensação de 'frio na barriga' & 0 & 1 & 2 & 3 \\
\hline Dor de estômago & 0 & 1 & 2 & 3 \\
\hline Estômago 'embrulhado' & 0 & 1 & 2 & 3 \\
\hline Vontade de urinar & 0 & 1 & 2 & 3 \\
\hline Sensação de boca seca & 0 & 1 & 2 & 3 \\
\hline Dificuldade para engolir & 0 & 1 & 2 & 3 \\
\hline Dor no pescoço & 0 & 1 & 2 & 3 \\
\hline Sensação de fraqueza nas pernas & 0 & 1 & 2 & 3 \\
\hline Contração ou tremor dos músculos & 0 & 1 & 2 & 3 \\
\hline Sensação de tensão na testa & 0 & 1 & 2 & 3 \\
\hline $\begin{array}{l}\text { Sensação de tensão nos músculos } \\
\text { da mandíbula ('músculos da mordida') }\end{array}$ & 0 & 1 & 2 & 3 \\
\hline \multicolumn{5}{|l|}{ Índice de depressão Zung modificado } \\
\hline \multicolumn{5}{|c|}{ Para cada uma das perguntas abaixo, indique o que melhor descreve como você se sente recentemente. } \\
\hline & $\begin{array}{l}\text { Raramente ou } \\
\text { muito pouco } \\
\text { (menos de } 1 \text { dia } \\
\text { por semana) }\end{array}$ & $\begin{array}{l}\text { Uma pequena } \\
\text { parte do tempo } \\
\text { (1-2 dias } \\
\text { por semana) }\end{array}$ & $\begin{array}{l}\text { Razoável parte } \\
\text { do tempo } \\
\text { ( } 3-4 \text { dias por } \\
\text { semana) }\end{array}$ & $\begin{array}{l}\text { A maior parte } \\
\text { do tempo } \\
\text { (5-7 dias por } \\
\text { semana) }\end{array}$ \\
\hline 1. Eu me sinto desanimado e triste & 0 & 1 & 2 & 3 \\
\hline 2. Eu me sinto melhor de manhã & 3 & 2 & 1 & 0 \\
\hline $\begin{array}{l}\text { 3. Tenho crises de choro } \\
\text { ou vontade de chorar }\end{array}$ & 0 & 1 & 2 & 3 \\
\hline 4. Tenho dificuldades para dormir à noite & 0 & 1 & 2 & 3 \\
\hline 5. Acho que ninguém se importa comigo & 0 & 1 & 2 & 3 \\
\hline $\begin{array}{l}\text { 6. Estou comendo mais do que } \\
\text { costumava comer }\end{array}$ & 3 & 2 & 1 & 0 \\
\hline 7. Eu ainda gosto de sexo & 3 & 2 & 1 & 0 \\
\hline 8. Percebo que estou perdendo peso & 0 & 1 & 2 & 3 \\
\hline $\begin{array}{l}\text { 9. Estou com problemas de } \\
\text { constipação (prisão de ventre) }\end{array}$ & 0 & 1 & 2 & 3 \\
\hline $\begin{array}{l}\text { 10. Meu coração está batendo mais } \\
\text { rápido do que o habitual }\end{array}$ & 0 & 1 & 2 & 3 \\
\hline 11. Fico cansado sem motivo & 0 & 1 & 2 & 3 \\
\hline
\end{tabular}


Tabela 2 (Continued)

\begin{tabular}{|l|l|l|l|l|}
\hline $\begin{array}{l}\text { 12. Tenho a mesma clareza } \\
\text { de ideias que antigamente }\end{array}$ & 3 & 2 & 1 & 0 \\
\hline $\begin{array}{l}\text { 13. Eu tenho tendência de } \\
\text { acordar muito cedo }\end{array}$ & 0 & 1 & 2 & 3 \\
\hline $\begin{array}{l}\text { 14. Tenho a mesma facilidade para fazer } \\
\text { as coisas que costumava }\end{array}$ & 3 & 1 & 1 & 0 \\
\hline 15. Estou agitado e não consigo ficar parado & 0 & 2 & 2 & 1 \\
\hline 16. Tenho esperança quanto ao meu futuro & 3 & 1 & 2 & 3 \\
\hline 17. Estou mais irritado do que o habitual & 0 & 3 & 1 & 0 \\
\hline 18. Acho fácil tomar decisões & 0 & 2 & 2 & 3 \\
\hline 19. Sinto-me bastante culpado & 3 & 2 & 1 & 0 \\
\hline 20. Acho que sou útil e necessário & 3 & 1 & 1 & 3 \\
\hline 21. Minha vida é bastante completa & 0 & 2 & 2 & 0 \\
\hline $\begin{array}{l}\text { 22. Acho que os outros estariam } \\
\text { melhor se eu estivesse morto }\end{array}$ & 3 & 1 & 0 \\
\hline $\begin{array}{l}\text { 23. Ainda sou capaz de gostar } \\
\text { das coisas que eu costumava }\end{array}$ & & 2 & 3 \\
\hline
\end{tabular}

Abreviação: MSPQ, questionário modificado de percepção somática.

Foi calculado o CCI entre a primeira e a segunda aplicações dos questionários MSPQ e Zung. Para o MSPQ, o CCI foi de $0,688$ ( $p=0,0001)$. Para Zung, foi de 0,659 $(p=0,0001)$.

\section{Discussão}

No que se refere à dor lombar inespecífica, os aspectos psicossociais desfavoráveis, como ansiedade, depressão ou compensação trabalhista, são preditores de incapacidade de curta e longa duração ${ }^{3}$ e fatores de risco para maus resultados de qualquer modalidade terapêutica, com frequência três a quatro vezes maior do que em indivíduos psicologicamente saudáveis. ${ }^{27}$ Assim, a assistência à dor lombar deve identificar fatores de mau prognóstico para a abordagem terapêutica adequada, ${ }^{16,18}$ através da aplicação de uma das ferramentas de triagem psicométrica disponíveis (DRAM, StarT Back, Orebro) e de um questionário de incapacidade (índice de Oswestry). ${ }^{28}$

Mesmo com a ampla literatura favorável ao uso rotineiro de ferramentas de avaliação psicológica, o número de médicos assistentes que se utilizam desses instrumentos na prática clínica é relativamente baixo. ${ }^{27,29}$ Segundo Daubs et $\mathrm{al}^{29}$ no entanto, cirurgiões de coluna são capazes de diagnosticar distúrbios psicológicos em apenas 16,9\% dos casos de indivíduos portadores de distúrbios psicológicos em tratamento. Mesmo assim, a maioria desses especialistas (63\%) não utiliza qualquer instrumento de avaliação psicométrica na prática cotidiana. ${ }^{27}$

O DRAM ${ }^{16}$ foi especificamente desenvolvido como ferramenta de triagem para casos de dor lombar no sistema de saúde britânico e consiste da soma das pontuações de dois questionários distintos: O MSPQ e o Zung, que avaliam estados de ansiedade e depressão, respectivamente, e classificam os indivíduos em quatro subtipos psicológicos distintos. No artigo original de Main, ${ }^{16}$ o autor relatou que os indivíduos do subtipo N submetidos a tratamento cirúrgico evoluíram de forma satisfatória e que a conversão dos subtipos R em subtipo $\mathrm{N}$ confirmava o resultado cirúrgico favorável. Quanto aos indivíduos submetidos a tratamento conservador, os do subtipo $\mathrm{R}$ apresentavam desfechos desfavoráveis duas vezes mais frequentemente que os subtipos $\mathrm{N}$, enquanto os subtipos DD e DS apresentavam três a quatro vezes mais chances de resultados insatisfatórios.

O DRAM já foi usado por inúmeros autores como ferramenta complementar na avaliação de resultados de cirurgia, ${ }^{29-33}$ assim como já destacado no estudo original. ${ }^{16}$ Os instrumentos Orebro e StarT Back, mais recentes, têm sido usados na literatura para estudos relacionados à triagem de tratamento conservador e aspectos psicossociais de portadores de dor na coluna. ${ }^{34,35}$ Comparado aos outros, o DRAM é mais extenso (45 questões) que o Orebro (23 questões) ${ }^{36}$ e o StarT Back (9 questões), ${ }^{9}$ característica indesejável para aplicação na prática cotidiana atual.

Os critérios para certificar a qualidade da versão final do DRAM português brasileiro foram consistência interna e reprodutibilidade. ${ }^{24} \mathrm{~A}$ consistência interna foi determinada pelo índice de Cronbach alfa e resultou em 0,815 para o MSPQe 0,794 para o Zung, os quais confirmam a qualidade da versão final do DRAM, dentro dos parâmetros sugeridos para questionários de qualidade de vida entre 0,70 e 0,90. ${ }^{24}$ Para estudo de reprodutibilidade, foi escolhido o $\mathrm{CCI}$, que resultou em valores de $0,688(p=0,0001)$ para o MSPQ e de 0,659 $(p=0,0001)$ para o Zung, que segundo Cicchetti, ${ }^{37}$ valores entre 0,60 e 0,74 são considerados suficientes para garantir a qualidade estatística das avaliações. Segundo os critérios estatísticos propostos, a versão final do DRAM em português brasileiro é confiável e reprodutível, além de equivalente à versão original, podendo ser utilizada na prática clínica.

Ainda que os resultados de confiabilidade e reprodutibilidade tenham atingido o objetivo inicial do presente estudo 
para garantir a qualidade da versão final do DRAM em português brasileiro, alguns critérios adicionais poderiam ter sido utilizados, especialmente o estudo de validade do construto em comparação a um questionário semelhante, como o Orebro. Esta foi uma limitação do presente estudo.

O DRAM foi desenvolvido em 1992, tendo sido o pioneiro dos questionários psicométricos desenvolvidos especificamente para um grupo de indivíduos portadores de dor lombar. Seu formato e extensão, portanto, não foram alvo da atenção dos seus idealizadores, mas certamente seu conteúdo e sua capacidade de triar perfis psicológicos favoráveis a diferentes modalidades de tratamento. Portanto, se comparado a questionários mais recentes, como o StarT Back, o DRAM é mais extenso e detalhado. Porém, na experiência do autor principal do presente estudo, ainda que mais longo, é aplicável aos pacientes no consultório privado ou de serviço público.

A tradução do DRAM para o português brasileiro abre perspectivas para futuras pesquisas: DRAM como ferramenta de desfecho; DRAM como ferramenta específica de seleção de pacientes para tratamento; desenvolvimento de outra ferramenta psicométrica originalmente para a população brasileira.

$\mathrm{O}$ uso de ferramentas psicométricas é um diferencial na atual abordagem às doenças degenerativas da coluna vertebral, especialmente naquelas em que pode haver solução cirúrgica, já que indivíduos portadores de diagnósticos idênticos, como radiculopatia lombar, por exemplo, mas com perfis psicológicos distintos, podem obter desfechos bastante diversos com tratamentos similares. ${ }^{38}$ Nesses casos, a compreensão mínima do estado psicológico de cada paciente capacita o cirurgião para a escolha terapêutica mais efetiva para cada indivíduo, especialmente naqueles em quem os perfis psicológicos favorecem abordagens conservadoras.

\section{Conclusão}

A versão do questionário DRAM em português brasileiro apresentada neste estudo é válida e está disponível para uso na prática clínica segundo os critérios adotados pela literatura aplicados ao presente estudo.

As pesquisas futuras devem aprofundar a compreensão da percepção de dor e o impacto das variantes psicológicas nas decisões terapêuticas e seus desfechos, além de buscar o desenvolvimento de instrumentos psicométricos precisos e individualizados para aplicação maciça. Ainda mais importante é o engajamento dos médicos na inclusão no arsenal de avaliação do paciente com sintoma da coluna das ferramentas psicométricas, além dos dados clínicos e de imagem na instituição da melhor terapêutica e na avaliação dos desfechos, principalmente.

Conflito de Interesses

Os autores declaram não haver conflito de interesses.

\section{Referências}

1 Ehrlich GE. Low back pain. Bull World Health Organ 2003;81(09): 671-676

2 Kikuchi S. New concept for backache: biopsychosocial pain syndrome. Eur Spine J 2008;17(Suppl 4):421-427
3 Carragee EJ, Alamin TF, Miller JL, Carragee JM. Discographic, MRI and psychosocial determinants of low back pain disability and remission: a prospective study in subjects with benign persistent back pain. Spine J 2005;5(01):24-35

4 Trief PM, Grant W, Fredrickson B. A prospective study of psychological predictors of lumbar surgery outcome. Spine 2000;25 (20):2616-2621

5 Ryan CG, Gray HG, Newton M, Granat MH. The relationship between psychological distress and free-living physical activity in individuals with chronic low back pain. Man Ther 2010;15(02):185-189

6 Trief PM, Ploutz-Snyder R, Fredrickson BE. Emotional health predicts pain and function after fusion: a prospective multicenter study. Spine 2006;31(07):823-830

7 Amaral V, Marchi L, Martim H, et al. Influence of psychosocial distress in the results of elective lumbar spine surgery. J Spine Surg 2017;3(03):371-378

8 Hill JC, Vohora K, Dunn KM, Main CJ, Hay EM. Comparing the STarT back screening tool's subgroup allocation of individual patients with that of independent clinical experts. Clin J Pain 2010;26(09): 783-787

9 Hill JC, Dunn KM, Main CJ, Hay EM. Subgrouping low back pain: a comparison of the STarT Back Tool with the Orebro Musculoskeletal Pain Screening Questionnaire. Eur J Pain 2010;14(01):83-89

10 Pilz B, Vasconcelos RA, Teixeira PP, et al. Construct and discriminant validity of STarT Back Screening Tool - Brazilian version. Braz J Phys Ther 2017;21(01):69-73

11 Johnsen LG, Hellum C, Nygaard OP, et al. Comparison of the SF6D, the EQ5D, and the oswestry disability index in patients with chronic low back pain and degenerative disc disease. BMC Musculoskelet Disord 2013;14:148

12 Falavigna A, Teles AR, Braga GL, Barazzetti DO, Lazzaretti L, Tregnago AC. Instrumentos de avaliação clínica e funcional em cirurgia da coluna vertebral. Coluna/Columna 2011;10(01): 62-67

13 Alhowimel A, AlOtaibi M, Radford K, Coulson N. Psychosocial factors associated with change in pain and disability outcomes in chronic low back pain patients treated by physiotherapist: A systematic review. SAGE Open Med 2018;6:2050312118757387

14 Wilhelm M, Reiman M, Goode A, et al. Psychological predictors of outcomes with lumbar spinal fusion: a systematic literature review. Physiother Res Int 2017;22(02):. Doi: 10.1002/pri.1648

15 Nicholls JL, Azam MA, Burns LC, et al. Psychological treatments for the management of postsurgical pain: a systematic review of randomized controlled trials. Patient Relat Outcome Meas 2018; 9:49-64

16 Main CJ, Wood PL, Hollis S, Spanswick CC, Waddell G. The Distress and Risk Assessment Method. A simple patient classification to identify distress and evaluate the risk of poor outcome. Spine 1992;17(01):42-52

17 Main CJ. The Modified Somatic Perception Questionnaire (MSPQ). J Psychosom Res 1983;27(06):503-514

18 Main CJ, Waddell G. The back pain revolution. In: Waddell G, ed. London: Churchill Livingstone; 1998

19 Gandek B, Ware JE Jr. Methods for validating and norming translations of health status questionnaires: the IQOLA Project approach. International Quality of Life Assessment. J Clin Epidemiol 1998;51(11):953-959

20 Beaton DE, Bombardier C, Guillemin F, Ferraz MB. Guidelines for the process of cross-cultural adaptation of self-report measures. Spine 2000;25(24):3186-3191

21 Nordin M, Randhawa K, Torres P, et al. The Global Spine Care Initiative: a systematic review for the assessment of spine-related complaints in populations with limited resources and in low- and middle-income communities. Eur Spine J 2018;27(Suppl 6):816-827

22 Berkanovic E. The effect of inadequate language translation on Hispanics' responses to health surveys. Am J Public Health 1980; 70(12):1273-1276 
23 Alanay A, Cil A, Berk H, et al. Reliability and validity of adapted Turkish Version of Scoliosis Research Society-22 (SRS-22) questionnaire. Spine 2005;30(21):2464-2468

24 Terwee CB, Bot SDM, de Boer MR, et al. Quality criteria were proposed for measurement properties of health status questionnaires. J Clin Epidemiol 2007;60(01):34-42

25 Pereira J. Análise de dados qualitativos: estratégias metodológicas para as ciências da saúde, humanas e sociais. 2a. ed. São Paulo: Edusp; 1999

26 Mehta S, Bastero-Caballero RF, Sun Y, et al. Performance of intraclass correlation coefficient (ICC) as a reliability index under various distributions in scale reliability studies. Stat Med 2018;37 (18):2734-2752

27 Grevitt M, Pande K, O'Dowd J, Webb J. Do first impressions count? A comparison of subjective and psychologic assessment of spinal patients. Eur Spine J 1998;7(03):218-223

28 Vigatto R, Alexandre NM, Correa Filho HR. Development of a Brazilian Portuguese version of the Oswestry Disability Index: cross-cultural adaptation, reliability, and validity. Spine 2007;32 (04):481-486

29 Daubs MD, Patel AA, Willick SE, et al. Clinical impression versus standardized questionnaire: the spinal surgeon's ability to assess psychological distress. J Bone Joint Surg Am 2010;92(18): 2878-2883

30 Vialle E, de Oliveira Pinto BM, Vialle LR, Gomez JD. Evaluation of psychosomatic distress and its influence in the outcomes of lumbar fusion procedures for degenerative disorders of the spine. Eur J Orthop Surg Traumatol 2015;25(01, Suppl 1):S25-S28
31 Pollock R, Lakkol S, Budithi C, Bhatia C, Krishna M. Effect of psychological status on outcome of posterior lumbar interbody fusion surgery. Asian Spine J 2012;6(03):178-182

32 Abtahi AM, Brodke DS, Lawrence BD, Zhang C, Spiker WR. Association between patient-reported measures of psychological distress and patient satisfaction scores after spine surgery. J Bone Joint Surg Am 2015;97(10):824-828

33 Theologis AA, Ailon T, Scheer JK, et al. International Spine Study Group. Impact of preoperative depression on 2-year clinical outcomes following adult spinal deformity surgery: the importance of risk stratification based on type of psychological distress. J Neurosurg Spine 2016;25(04):477-485

34 Soer R, Vroomen P, Stewart R, et al. Groningen Spine Study Group. Factor analyses for the Örebro Musculoskeletal Pain Questionnaire for working and nonworking patients with chronic low back pain. Spine J 2017;17(04):603-609

35 Fjeld O, Grotle M, Siewers V, Pedersen LM, Nilsen KB, Zwart JA. Prognostic Factors for Persistent Leg-Pain in Patients Hospitalized With Acute Sciatica. Spine 2017;42(05):E272-E279

36 Linton SJ, Halldén K. Can we screen for problematic back pain? A screening questionnaire for predicting outcome in acute and subacute back pain. Clin J Pain 1998;14(03):209-215

37 Cicchetti D. Guidelines, criteria, and rules of thumb for evaluating normed and standardized assessment instruments in psychology. Psychol Assess 1994;6(04):284-290

38 Daubs MD, Norvell DC, McGuire R, et al. Fusion versus nonoperative care for chronic low back pain: do psychological factors affect outcomes? Spine 2011;36(21, Suppl)S96-S109 